\title{
The gemcitabine, docetaxel, and capecitabine (GTX) regimen for metastatic pancreatic cancer: a retrospective analysis
}

\author{
Robert L. Fine · David R. Fogelman · Stephen M. Schreibman · Manisha Desai · \\ William Sherman $\cdot$ James Strauss $\cdot$ Susan Guba $\cdot$ Riolan Andrade $\cdot$ John Chabot
}

Published online: 9 September 2007

(c) Springer-Verlag 2007

\section{Erratum to: Cancer Chemother Pharmacol DOI 10.1007/s00280-007-0473-0}

Erlotinib was erroneuosly included in the list of chemotherapeutic agents in the second sentence of paragraph 4 in Discussion. The first two sentences of this paragraph should thus read:

Many chemotherapeutic agents have been used in over 12 separate phase II or III doublet combinations with gemcitabine, many of which went on to become negative

The online version of the original article can be found under doi:10.1007/s00280-007-0473-0.

\section{R. L. Fine · D. R. Fogelman · W. Sherman}

Experimental Therapeutics Program,

Division of Medical Oncology, Department of Medicine,

New York Presbyterian Medical Center,

Columbia University College of Physicians

and Surgeons, and The Pancreas Center at Columbia,

New York, NY, USA

\section{S. M. Schreibman}

Oncology and Hematology Specialists,

Morristown Memorial Hospital, Morristown, NJ, USA

\section{Desai}

Department of Biostatistics, Mailman School of Public Health, New York Presbyterian Medical Center,

Columbia University College of Physicians and Surgeons, and The Pancreas Center at Columbia, New York, NY, USA

J. Strauss

U.S. Oncology Associates, Dallas, TX, USA trials in large phase III studies in pancreatic cancer. These included oxaliplatin [21], cisplatin [22], irinotecan [23] and capecitabine $[10,16]$.

S. Guba

Texas Cancer Associates, Dallas, TX, USA

R. Andrade

Hematology-Oncology Associates,

Poughkeepsie, NY, USA

J. Chabot

Whipple Service, Department of Surgery,

New York Presbyterian Medical Center,

Columbia University College of Physicians and Surgeons, and The Pancreas Center at Columbia, New York, NY, USA

R. L. Fine ( $\square)$

Division of Medical Oncology,

Columbia University Medical Center, 650 West 168th Street, Room 20-05,

New York, NY 10032, USA

e-mail: rlf20@columbia.edu 